\title{
THE STRESS-ENERGY TENSOR IN ELECTROMAGNETIC THEORY AND A NEW LAW OF FORCE.
}

\author{
By H. Bateman.
}

SyNOPSIS.

\begin{abstract}
Modified Electromagnetic Theory.-By suitably modifying the equations for the components of the stress-energy tensor it is possible to reconcile electromagnetic theory with the idea of non-radiating electronic orbits. The change, however, is equivalent to assuming that an element of electricity is acted upon by a new force which depends on the gradient of the density of electricity and balances the usual electromagnetic force. By assuming a certain distribution of density within an electron it is also possible to account for the existence of discrete electronic charges.
\end{abstract}

I. The Stress-energy Tensor.-We shall assume that the stress-energy tensor associated with a system of electric charges has the following components:

$$
\begin{aligned}
& W=\frac{1}{2}\left(E^{2}+H^{2}\right)+\frac{2}{c^{2}} \psi \frac{\partial^{2} \psi}{\partial t^{2}}+\left(\frac{\partial \psi}{\partial x}\right)^{2}+\left(\frac{\partial \psi}{\partial y}\right)^{2}+\left(\frac{\partial \psi}{\partial z}\right)^{2}-\frac{\mathbf{I}}{c^{2}}\left(\frac{\partial \psi}{\partial t}\right)^{2} ; \\
& S_{x}=c\left[E_{y} H_{z}-E_{z} H_{y}\right]-2 \psi \frac{\partial^{2} \psi}{\partial x \partial t}, \quad \text { etc.; } \\
& X_{x}=\frac{1}{2}\left[E_{x}^{2}-E_{y}^{2}-E_{z}^{2}+H_{x}^{2}-H_{y}^{2}-H_{z}^{2}\right] \\
& -2 \psi \frac{\partial^{2} \psi}{\partial x^{2}}+\left(\frac{\partial \psi}{\partial x}\right)^{2}+\left(\frac{\partial \psi}{\partial y}\right)^{2}+\left(\frac{\partial \psi}{\partial z}\right)^{2}-\frac{\mathrm{I}}{c^{2}}\left(\frac{\partial \psi}{\partial t}\right)^{2} \\
& Y_{x}=X_{y}=E_{x} E_{y}+H_{x} H_{y}-2 \psi \frac{\partial^{2} \psi}{\partial x \partial y}, \quad \text { etc. }
\end{aligned}
$$

The quantity $W$ represents the density of energy, the vector $S$ specifies the flow of energy and is proportional to the density of momentum; the six quantities $X_{x}, Y_{y}, Z_{z}, Y_{z}, Z_{x}, X_{y}$ are the components of stress; $v$ is the velocity of electricity at a point $(\xi, \eta, \zeta)$ where the density of electricity at time $\tau$ is $\rho$; $E$ and $H$ are the field vectors in the electromagnetic field produc $\mathrm{d}$ by the electric charges and $\psi$ is a retarded potential defined by the equations

$$
\begin{aligned}
& \psi=\frac{\mathrm{I}}{4 \pi} \mathcal{J} \frac{d \xi d \eta d \zeta}{r}\left[\rho \sqrt{\mathrm{I}-\frac{v^{2}}{c^{2}}}\right], \\
& r^{2}=(x-\xi)^{2}+(y-\eta)^{2}+(z-\zeta)^{2}=c^{2}(t-\tau)^{2}, \quad \tau<t,
\end{aligned}
$$

$c$ being the velocity of light. 
The components of the total force $F$ are given by the three equations of type

$$
F_{x}=\frac{\partial X_{x}}{\partial x}+\frac{\partial Y_{x}}{\partial y}+\frac{\partial Z_{x}}{\partial z}-\frac{\mathrm{I}}{c^{2}} \frac{\partial S_{x}}{\partial t}
$$

and we find that

$$
\begin{aligned}
F_{x} & =\rho\left[E_{x}+\frac{\mathrm{I}}{c}\left(v_{y} H_{z}-v_{z} H_{y}\right)\right]-2 \psi \frac{\partial}{\partial x}\left[\frac{\partial^{2} \psi}{\partial x^{2}}+\frac{\partial^{2} \psi}{\partial y^{2}}+\frac{\partial^{2} \psi}{\partial z^{2}}-\frac{\mathrm{I}}{c^{2}} \frac{\partial^{2} \psi}{\partial t^{2}}\right] \\
& =\rho\left[E_{x}+\frac{\mathrm{I}}{c}\left(v_{y} H_{z}-v_{z} H_{y}\right)\right]+2 \psi \frac{\partial}{\partial x}\left[\rho \sqrt{\mathrm{I}-\frac{v^{2}}{c^{2}}}\right] .
\end{aligned}
$$

The energy equation

$$
\frac{\partial S_{x}}{\partial x}+\frac{\partial S_{y}}{\partial y}+\frac{\partial S_{z}}{\partial z}+\frac{\partial W}{\partial t}=-(v \cdot F)
$$

requires that

where

$$
\psi \frac{d}{d t}\left[\rho \sqrt{\mathrm{I}-\frac{v^{2}}{c^{2}}}\right]=0
$$

$$
\frac{d}{d t}=\frac{\partial}{\partial t}+v_{x} \frac{\partial}{\partial x}+v_{y} \frac{\partial}{\partial y}+v_{z} \frac{\partial}{\partial z} .
$$

It seems reasonable to adopt the hypothesis that $F$ is everywhere zero.

2. The Existence of Discrete Charges.-When all the electric charges are stationary $\psi$ becomes the electrostatic potential and

$$
F_{x}=-\rho \frac{\partial \psi}{\partial x}+2 \psi \frac{\partial \rho}{\partial x} .
$$

consequently $F$ vanishes everywhere if

and this means that

$$
\begin{aligned}
& \rho \frac{\partial \psi}{\partial x}=2 \psi \frac{\partial \rho}{\partial x}, \\
& \rho \frac{\partial \psi}{\partial y}=2 \psi \frac{\partial \rho}{\partial y}, \\
& \rho \frac{\partial \psi}{\partial z}=2 \psi \frac{\partial \rho}{\partial z},
\end{aligned}
$$

$$
\psi=b^{2} \rho^{2},
$$

where $b^{2}$ is a constant. The function $\psi$ then satisfies the partial differential equation

$$
\frac{\partial^{2} \psi}{\partial x^{2}}+\frac{\partial^{2} \psi}{\partial y^{2}}+\frac{\partial^{2} \psi}{\partial z^{2}}+\frac{\mathbf{I}}{b} \sqrt{\psi}=0 .
$$




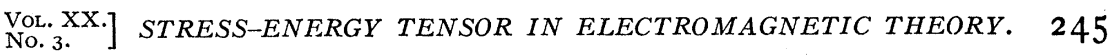

Let us consider the case in which $\rho$ is a function of the radius $r$ from a point $o$ and differs from zero only within a sphere of radius $a$. Assuming that

we find that

$$
\rho=A_{0}+A_{2}\left(\frac{r}{a}\right)^{2}+A_{4}\left(\frac{r}{a}\right)^{4}+\cdots,
$$

$\psi=\frac{\mathrm{I}}{3 \cdot 2}\left(3 a^{2}-r^{2}\right) A_{0}+\frac{\mathrm{I}}{4 \cdot 5}\left(5 a^{2}-\frac{r^{4}}{a^{2}}\right) A_{2}+\frac{\mathrm{I}}{6 \cdot 7}\left(7 a^{2}-\frac{r^{6}}{a^{4}}\right) A_{4}+\cdots ;$

consequently

$$
\begin{aligned}
b^{2}\left[A_{0}+A_{2}\left(\frac{r}{a}\right)^{2}+A_{4}\right. & \left.\left(\frac{r}{a}\right)^{4}+\cdots\right]^{2} \\
& =\frac{\mathrm{I}}{2 \cdot 3}\left(3 a^{2}-r^{2}\right) A_{0}+\frac{\mathrm{I}}{4 \cdot 5}\left(5 a^{2}-\frac{r^{4}}{a^{2}}\right) A_{2}+\cdots
\end{aligned}
$$

Equating coefficients we find that

$$
\begin{aligned}
& \rho=A_{0}\left[\mathrm{I}-\frac{\mathrm{I}}{\mathrm{I} 2} \frac{r^{2}}{A_{0} b^{2}}-\frac{\mathrm{I}}{720} \frac{r^{4}}{A_{0}^{2} b^{4}}-\frac{\mathrm{I}}{\mathrm{I} 0080} \frac{r^{6}}{A_{0}^{3} b^{6}}-\frac{3 \mathrm{I}}{6 ! 7 !} \frac{r^{8}}{A_{0}^{4} b^{8}}\right. \\
& \left.-\frac{(97 \mathrm{I})(24)}{(6 !)(\mathrm{II} !)} \frac{r^{10}}{A_{0}^{5} b^{10}}-\cdots\right] \text {, } \\
& f(\xi) \equiv \mathrm{I}-\frac{\mathrm{I}}{2} \xi+\frac{\mathrm{I}}{48} \xi^{2}+\frac{\mathrm{I}}{4320} \xi^{3}+\frac{\mathrm{I}}{80640} \xi^{4}+\frac{3 \mathrm{I}}{(\mathrm{IO})(6 !)(7 !)} \xi^{5} \\
& +\frac{(97 \mathrm{I})(24)}{(6 !)(\mathrm{I} 2 !)} \xi^{6}+\cdots .
\end{aligned}
$$

The equation $f(\xi)=0$ has two roots, one of which is approximately $2 \cdot 2$; the second is difficult to determine accurately as it is necessary to consider a large number of terms of the series; it appears to lie between 12 and 6 and is probably about II.

When $a$ and the total charge $e$ are given, $A_{0}$ and $b$ are determined, but there are two possible solutions and so there are two different types of discrete charge. ${ }^{1}$ The present analysis does not, however, indicate the size of the electron.

1 It is interesting to compare the solution of the equation $\psi=b^{2} \rho^{2}$ with that of the simpler equation $\psi=b^{2} \rho$ which would also lead to discrete charges. In the latter case we may have

$$
\psi=\frac{\mathrm{I}}{r} \sin \left(\frac{r}{b}\right)=b^{2} \rho,
$$

the radius of the sphere being determined by the equation $a=b \pi\left(n+\frac{1}{2}\right)$, where $n$ is an integer. We have in fact

$$
\psi=\int_{r}^{a} \rho r d r+\frac{\mathrm{I}}{r} \int_{0}^{r} \rho r^{2} d r=\frac{\mathrm{I}}{b^{2}} \int_{r}^{a} \sin \left(\frac{r}{b}\right) d r+\frac{\mathrm{I}}{b^{2} r} \int_{0}^{r} \sin \left(\frac{r}{b}\right) r d r=\frac{\mathrm{I}}{r} \sin \left(\frac{r}{b}\right) .
$$

The total electric charge is then

$$
e=\int_{0}^{a} \rho r^{2} d r=\sin \left(n+\frac{1}{2}\right) \pi=(-\mathrm{r})^{n}
$$


3 The Radiation of Energy from a Moving Electric Pole.-In the case of a moving point charge of electricity we must put

$$
\psi=-\frac{e}{4 \pi M} \sqrt{c^{2}-\xi^{\prime 2}-\eta^{\prime 2}-\zeta^{\prime 2}}=-\frac{e}{4 \pi M} \sqrt{c^{2}-v^{2}},
$$

where $\xi(\tau), \eta(\tau), \zeta(\tau)$ are the coördinates of the charge $e$ at time $\tau$ and $M$ is defined as a function of $x, y, z, t$ by the equations

$$
\begin{gathered}
M=[x-\xi(\tau)] \xi^{\prime}(\tau)+[y-\eta(\tau)] \eta^{\prime}(\tau)+[z-\zeta(\tau)] \zeta^{\prime}(\tau)-c^{2}(t-\tau) \\
{[x-\xi(\tau)]^{2}+[y-\eta(\tau)]^{2}+[z-\zeta(\tau)]^{2}=c^{2}(t-\tau)^{2}} \\
\tau<t .
\end{gathered}
$$

Calculating $S_{x}$ and retaining only terms of order I $/ M^{2}$ we have

$$
\begin{aligned}
S_{x} & \sim \frac{e^{2} c^{2}(t-\tau)(x-\xi)}{\mathrm{I} 6 \pi^{2} M^{4}}\left[\left\{v^{\prime 2}-2\left(v v^{\prime}\right) \frac{K}{M}-\left(c^{2}-v^{2}\right) \frac{K^{2}}{M^{2}}\right\}\right. \\
& \left.+6\left(v v^{\prime}\right) \frac{K}{M}+6\left(c^{2}-v^{2}\right) \frac{K^{2}}{M^{2}}-2 v^{\prime 2}-\frac{2\left(v v^{\prime}\right)^{2}}{c^{2}-v^{2}}-2\left(v v^{\prime \prime}\right)-\frac{2 L}{M}\left(c^{2}-v^{2}\right)\right],
\end{aligned}
$$

where

$$
\begin{aligned}
& K=(x-\xi) \xi^{\prime \prime}+(y-\eta) \eta^{\prime \prime}+(z-\zeta) \xi^{\prime \prime}, \\
& L=(x-\xi) \xi^{\prime \prime \prime}+(y-\eta) \eta^{\prime \prime \prime}+(z-\zeta) \xi^{\prime \prime \prime} .
\end{aligned}
$$

To calculate the radiation we must first of all replace $(x-\xi)$ in $S_{x}$ by $c(t-\tau)$ to get the radial component of $S$. We must next multiply by a Doppler factor and this ${ }^{1}$ is equivalent to replacing $(x-\xi)$ in $S_{x}$ by $-M / c$. The final step is to integrate over a large sphere whose center is at the pole. Writing

$$
I_{n}^{m}=\int_{0}^{\pi} \frac{\cos ^{m} \theta \sin \theta d \theta}{(c-v \cos \theta)^{n}}
$$

we have

$$
\begin{array}{ll}
I_{3}{ }^{0}=\frac{2 c}{\left(c^{2}-v^{2}\right)^{2}}, & I_{4}{ }^{1}=\frac{8 c v}{3\left(c^{2}-v^{2}\right)^{3}}, \\
I_{5}{ }^{0}=\frac{2 c\left(c^{2}+v^{2}\right)}{\left(c^{2}-v^{2}\right)^{4}}, & I_{5}{ }^{2}=\frac{2 c\left(c^{2}+5 v^{2}\right)}{3\left(c^{2}-v^{2}\right)^{4}},
\end{array}
$$

and there is a different type of discrete charge for each positive integral value of $n$. In this case $\rho$ is determined by a homogeneous linear integral equation while in the other case the integral equation is not linear. The equation $\psi=b^{2} \rho$ is used by A. Korn in his mechanical theory of the electromagnetic field. Phys. Zeitschr., Bd. I9 (I9I8), p. 234. Another distribution of density is considered by G. Mie, Ann. d. Phys., Bd. 39 (Igr2), p. r.

${ }^{1}$ Cf. M. Abraham, Theorie der Elektrizität, Bd. II., p. I08. A Liénard, L'éclairage électrique (I898). 
and we find that the rate of radiation is

$$
\begin{aligned}
R & =\frac{e^{2}}{6 \pi c^{3}}\left[\frac{\dot{v}^{2}+(v \ddot{v})}{\left(c^{2}-v^{2}\right)^{2}}+\frac{4(v \dot{v})^{2}}{\left(c^{2}-v^{2}\right)^{3}}\right] \\
& =\frac{e^{2}}{6 \pi c^{3} d \tau}\left[\frac{(v \dot{v})}{\left(c^{2}-v^{2}\right)^{2}}\right]=\frac{e^{2}}{\mathrm{I} 2 \pi c^{3}} \frac{d^{2}}{d \tau^{2}}\left[\frac{\mathrm{I}}{c^{2}-v^{2}}\right] .
\end{aligned}
$$

In a periodic motion the integral

\section{$\int R d \tau$}

taken over a complete period is zero and so there is no radiation of energy to infinity on the average.

It may be remarked that there is no radiation of energy to infinity when the components of the stress-energy tensor are given by ${ }^{1}$

$$
\begin{aligned}
& W=\left(\frac{\partial \psi}{\partial x}\right)^{2}+\left(\frac{\partial \psi}{\partial y}\right)^{2}+\left(\frac{\partial \psi}{\partial z}\right)^{2}+\frac{\mathrm{I}}{c^{2}}\left(\frac{\partial \psi}{\partial t}\right)^{2}-\frac{\mathrm{I}}{2}\left(E^{2}+H^{2}\right), \\
& S_{x}=-2 \frac{\partial \psi}{\partial t} \frac{\partial \psi}{\partial x}-c\left(E_{y} H_{z}-E_{z} H_{y}\right) \\
& X_{x}=-\left(\frac{\partial \psi}{\partial x}\right)^{2}+\left(\frac{\partial \psi}{\partial y}\right)^{2}+\left(\frac{\partial \psi}{\partial z}\right)^{2}+\frac{\mathrm{I}}{c^{2}}\left(\frac{\partial \psi}{\partial t}\right)^{2} \\
& -\frac{\mathrm{I}}{2}\left(E_{x}{ }^{2}-E_{y}{ }^{2}-E_{z}{ }^{2}\right)-\frac{\mathrm{I}}{2}\left(H_{x}{ }^{2}-H_{y}{ }^{2}-H_{z}{ }^{2}\right), \\
& X_{y}=Y_{x}=-2 \frac{\partial \psi}{\partial x} \frac{\partial \psi}{\partial y}-E_{x} E_{y}-H_{x} H_{y},
\end{aligned}
$$

but in this case the force is

$$
F_{x}=2 \rho \sqrt{\mathrm{I}-\frac{v^{2}}{c^{2}}} \frac{\partial \psi}{\partial x}-\rho\left[E_{x}+\frac{\mathrm{I}}{c}\left(v_{y} H_{z}-v_{z} H_{y}\right)\right]
$$

and cannot be made to vanish at each point.

The present analysis does not lead to quantum theory because the electron has been treated as a point charge. It looks as if certain conditions may have to be satisfied in order that $F$ may vanish everywhere when an electron of finite size describes an orbit round a positive nucleus. A discussion of this problem with the aid of the equations of $\S$ I looks difficult and will not be attempted here.

California Institute of Technology, Pasadena, April ro, r922.

${ }_{1}^{1}$ The expressions depending on $\psi$ are similar to those used by Volterra, Abraham and McLaren in the theory of gravitation. V. Volterra, Il Nuovo Cimento (I899), p. 337; M. Abraham, Phys Zeitschr. (I9I2), p. I; S. B. McLaren, Phil. Mag., Oct., I913, p. 636. 
NOTE, ADDED JUne 9, 1922.

It may be shown also that, when a point charge of electricity moves with an arbitrary velocity less than $c$, the rate at which linear momentum is radiated to infinity is the time derivative of a uniform function of the velocity and acceleration of the point charge. There is no radiation of momentum in a certain interval of time if the velocity and acceleration have the same values at the beginning and end of the interval.

With the new stress-energy tensor there appears also to be no radiation to infinity of angular momentum in the field of a Hertzian dipole.

Negative values of $W$ may be avoided by introducing an additional stress-energy tensor corresponding to a universal constant negative pressure. 